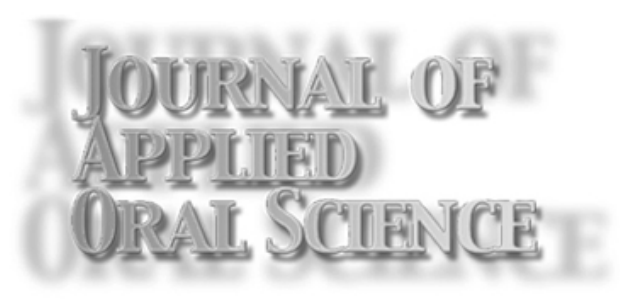

ISSN 1678-7757

\title{
Editorial
}

\section{The Cochrane Collaboration and Oral Health: a Brazilian experience}

The importance of systematic reviews as reliable sources of evidence for clinical practice is increasingly acknowledged worldwide. The Cochrane Collaboration is an international organization dedicated to improving health care for the world's population by preparing, maintaining and promoting the accessibility of its reviews of the evidence of the effects of healthcare interventions. Cochrane Reviews are needed to help ensure that healthcare decisions throughout the world can be informed by high quality and timely research evidence. Through a clearly defined process these reviews provide a logical synthesis and impartial summary of the existing research evidence. In addition they represent one of the best opportunities for dental clinicians to understand and accurately apply the current best evidence, which has been assembled from a methodologically robust and comprehensive search for solutions to dental problems.

The Cochrane Oral Health Group (OHG) was established in 1994 and is currently based at the School of Dentistry, The University of Manchester, United Kingdom. The range of systematic reviews within the remit of the OHG includes oral healthcare interventions covering prevention, treatment and health promotion. The level of international interest in the Cochrane OHG is illustrated by the diversity of its 620 members of whom 350 reviewers are from 40 different countries.

Training of Cochrane review authors is generally conducted either 'on line' through resources and materials available at (http:/ /www.cochrane.org/), or through regional workshops held by Cochrane entities. One of these workshops, "Evidence Based Medicine - a Cochrane Experience", was held at Universidade de São Paulo, Faculdade de Odontologia de Ribeirão Preto (FORP), in October 2007. Members of the Bahrain Branch of the UK Cochrane Centre in conjunction with colleagues from the Iberoamerican Cochrane Centre, the Iranian Cochrane Informal Network ICI Net and the Cochrane Developing Countries Network $(\mathrm{CDCN})$ delighted a Brazilian group of researchers. The facilitators at the workshop included Zbys Fedorowicz, Mona Nasser, Trent Outhouse and Jordi Pardo as well as colleagues from FORP.

In addition to several lectures, which aimed at raising awareness of Cochrane systematic reviews, training was provided on the identification and assessment of the methodological quality of randomized controlled trials. Prior to this workshop the Journal of Applied Oral Science had been registered on the Cochrane Collaboration US Cochrane Center's Masterlist of journals and during the workshop every issue of JAOS was handsearched for reports of randomized controlled trials. Citations and abstracts of these trials were subsequently delivered to the Cochrane Oral Health Group for uploading into The Cochrane Library.
During the three days of the workshop, the participants shared experiences and knowledge and had the opportunity to form various teams and to start preparing systematic reviews. Since this workshop several of the participants have registered Cochrane review titles and currently have four review protocols in the editorial process: Antibacterial agents in composite restorations to prevent dental caries; Interventions for cleaning dentures; Interventions for treating temporomandibular joint osteoarthritis; Interventions for the management of external root resorption, in addition to one completed review; Hand and ultrasonic instrumentation for orthograde root canal treatment of permanent teeth, which will be published in the Cochrane Database of Systematic Reviews in July 2008 .

These meetings in addition to a further workshop, which was held in Ribeirão Preto in February, were the initiative of, and organized by, Prof. Vinicius Pedrazzi in association with Prof. Raphael F. de Souza and Prof. Maximiliano S. Cenci. Additional training workshops including Cochrane protocol development and REVMAN 5 training are planned for the near future.

This group of researchers has also established a Google Group, which is used to share information about their activities, including further events aimed to bring together other interested colleagues and to raise awareness of the work of the Cochrane Oral Health Group throughout Brazil. The new teams created through this workshop are now organizing a local event to disseminate information about the Collaboration. For those interested in further information, please contact brohgcochrane@googlegroups.com for further information.

Free access to the Cochrane Library, which contains the Cochrane Database of Systematic Reviews (CDSR) and related materials, is provided to Latin America via: http://cochrane.bvsalud.org/.

Raphael F. Souza, DDS, PhD, Assistant Professor, University of São Paulo, Ribeirão Preto Dental School

Zbys Fedorowicz, MSc DPH, BDS, LDS RCS (Eng) Director, The Bahrain Branch of the UK Cochrane Centre, The Cochrane Collaboration

Carlos F. Santos, DDS, PhD, Associate Professor, Editor-in-Chief, Journal of Applied Oral Science 